\title{
The prognostic value of serum amyloid A in solid tumors: a meta-analysis
}

\author{
Hai-yingjie Lin ${ }^{1 \dagger}$, Guo-qiang Tan ${ }^{2 \dagger}$, Yan Liu ${ }^{3}$ and Shao-qiang Lin ${ }^{4 *}$
}

\begin{abstract}
Background: Previous studies have demonstrated that serum amyloid A (SAA) levels are correlated with the clinical outcomes of solid tumors. However, the available data have not been systematically evaluated. The objective of the present meta-analysis was to explore the prognostic value of SAA levels in solid tumors.

Methods: Eligible studies were identified from the PubMed, EMBASE and Science Citation Index electronic databases. The clinical characteristics, disease/progression-free survival (DFS/PFS) and overall survival (OS) were extracted from the eligible studies. The pooled hazard ratios (HRs) and 95\% confidence intervals (Cls) were calculated with Stata 12.0 software. We also performed subgroup, meta-regression and sensitivity analyses.

Results: In total, 12 eligible studies including 2749 patients were enrolled in the present meta-analysis. The pooled HRs with $95 \% \mathrm{Cl}$ s showed that elevated levels of SAA were significantly associated with poor OS ( $\mathrm{HR}=3.01,95 \% \mathrm{Cl}$ 1.96-4.63) and DFS/PFS (HR=1.67, 95\% Cl 1.31-2.12) in patients with solid tumors. Although publication bias was seem found in the studies with regard to OS, a further trim and fill analysis revealed that the adjusted HR was 3.02 (95\% Cl 1.96-4.63), which was close to the original HR. Subgroup analysis confirmed an elevated level of SAA as a strong prognostic marker in patients with solid tumors, regardless of tumor type, detection method, cut-off value, sample size, area and variance analyses.
\end{abstract}

Conclusion: Our meta-analysis indicated that elevated levels of SAA might be an unfavorable prognostic marker for OS in patients with solid tumors.

Keywords: Serum amyloid A, Solid tumors, Prognosis, Meta-analysis

\section{Introduction}

Despite the efforts of the scientific community, cancer is still a serious public health problem worldwide. Based on GLOBOCAN estimates, approximately 14.1 million new cancer cases and 8.2 million cancer-related deaths occurred in 2012 worldwide [1]. Early diagnosis and treatment monitoring can improve the prognosis of cancer patients. However, most serum biomarkers are lack of sensitivity and specificity for cancer patients in early or localized disease [2]. Therefore, there is urgent need to

\footnotetext{
*Correspondence: sqlin123@163.com

${ }^{\dagger}$ Hai-Yingjie Lin and Guo-qiang Tan contributed equally to this work

${ }^{4}$ Clinical Department of Guangdong Metabolic Disease Research

Center of Integrated Chinese and Western Medicine, The First Affiliated Hospital of Guangdong Pharmaceutical University, Guangzhou 510080,

Guangdong, China

Full list of author information is available at the end of the article
}

identify a novel biomarker that can effectively monitor progression and predict prognosis in cancer patients.

Previous studies have proposed the concept that chronic inflammation promotes cancer development and progression $[3,4]$. It has been widely accepted that tumor microenvironment is largely influenced by various inflammatory cells, which are key mediators of tumor growth, progression, and angiogenesis and metastasis [5]. Serum amyloid A (SAA) is an acute-phase, hepatic protein secreted in the course of acute infections and tissue damage, the expression of which is induced by several cytokines, including IL-1, IL-6, and tumor necrosis factor- $\alpha$ [6]. Research has demonstrated that the level of SAA may rapidly increase by up to 1000 -fold in response to acute inflammation, and it is an ideal marker for inflammation in the body [7]. Furthermore, an elevated protein level of SAA protein is observed in cancer

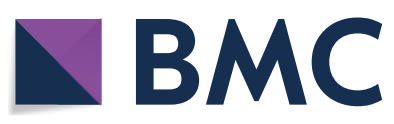

(c) The Author(s) 2019. This article is distributed under the terms of the Creative Commons Attribution 4.0 International License (http://creativecommons.org/licenses/by/4.0/), which permits unrestricted use, distribution, and reproduction in any medium, provided you give appropriate credit to the original author(s) and the source, provide a link to the Creative Commons license, and indicate if changes were made. The Creative Commons Public Domain Dedication waiver (http://creativecommons.org/ publicdomain/zero/1.0/) applies to the data made available in this article, unless otherwise stated. 
patients at an early stage, this finding has been identified both by immunochemistry and by proteomics methods in different common cancers, such as lung, ovarian, renal, uterine, nasopharyngeal cancer and in melanoma [8]. These results revealed that SAA may be as a potentially useful biomarker for cancer.

Meta-analysis has shown that a high level of C-reactive protein (CRP), another acute-phase protein, is significantly associated with the poor prognosis of some cancers, including esophageal, colorectal and urological cancers, and it has already been reported to be a prognostic marker in relevant cancers [9-11]. Latest meta-analysis suggests that high SAA levels were closely associated with a risk of developing cancer risk, but not to confirm their relation in terms of prognosis [12]. However, to the best of our knowledge, no meta-analysis has explored the prognostic value of SAA in cancer patients. Therefore, we performed the current quantitative metaanalysis to identify the prognostic significance of SAA levels in human solid tumors. These results will provide important information for personalized therapy.

\section{Materials and methods Search strategy}

A comprehensive search strategy was employed to search PubMed, EMBASE and Science Citation Index up to February 2019 without applying a start date limit. The terms neoplasms, serum amyloid A protein, prognosis and cohort studies were used as medical subject headings $(\mathrm{MeSH})$ and key words at the same time.

\section{Inclusion and exclusion criteria}

Eligible studies in this meta-analysis met the following inclusion criteria: (1) patients were pathologically diagnosed with any type of solid tumors. (2) SAA as isolated from serum samples. (3) The study was designed as a cohort study. (4) The hazard ratios (HRs) with 95\% confidence intervals (CIs) for survival outcomes were reported or could be calculated from the available data in the study.

The exclusion criteria were as follows: (1) the study did not report the prognostic value of SAA in solid tumors. (2) The study had a small sample size, with fewer than 50 patients. (3) The study was a case report, letter, conference abstract, review or duplicate article. (4) The article was not written in English.

\section{Quality assessment}

Two independent reviewers separately assessed the quality of the studies using the Newcastle-Ottawa Scale (NOS), which addresses three aspects, namely, patient selection, study comparability and study endpoints. The maximum possible NOS score is 9. Studies that earned scores $\geq 5$ were considered high quality, otherwise, they was considered low quality and removed. Any disagreement in the quality assessment of studies was settled by discussion.

\section{Data extraction and statistical analysis}

The following information was extracted: surname of the first author, publication year, country, tumor types, clinical stage, patient number, methods of SAA detection, cut-off value, duration of follow-up, outcome, and variance analysis. Furthermore, HRs and $95 \%$ CIs were obtained directly from the eligible studies or estimated using the method suggested by Tierney et al. [13]. The above information was collected by two independent researchers, and any disagreement was resolved by group discussion and consensus.

All statistical analyses in the present meta-analysis were conducted using Stata 12.0 software. Pooled HRs and $95 \%$ CIs were used to evaluate the prognostic value of SAA levels in solid tumors. When the pooled HR was greater than 1, we concluded that an elevated level of SAA was a negative prognostic factor for patients. The heterogeneity of the pooled results was measured using Cochran's $Q$ test and the $I^{2}$ statistic. Significant heterogeneity was defined as $P<0.1$ or $I^{2}>50 \%$. The random effects model was chosen to investigate the pooled HR when significant heterogeneity existed. Otherwise, the fixed effects model was used. To assess whether the results were influenced by other factors, subgroup, metaregression and sensitivity analyses were conducted. Publication bias was tested by the funnel plot and Begg's and Egger's tests. If publication bias was found, a trim and fill analysis was used to evaluate the number of missing studies and recalculate the pooled risk estimate with the addition of those missing studies [14].

\section{Results}

\section{Study selection}

In total, 261 articles were initially collected by a systematic literature search of the PubMed, EMBASE and Science Citation Index electronic databases. By reading the titles and author details, 183 articles were excluded due to not involving SAA or being duplicate articles. The remaining 78 articles were further evaluated by inspecting the abstracts, and 60 articles were removed according to the exclusion criteria. In total, 18 studies were assessed by reading the full text, after which 6 studies were excluded because HRs could not be obtained from them. Finally, 12 eligible articles were included in this meta-analysis. The flow chart of the study selection process is shown in Fig. 1. 
Studies acquired from database $(n=261)$

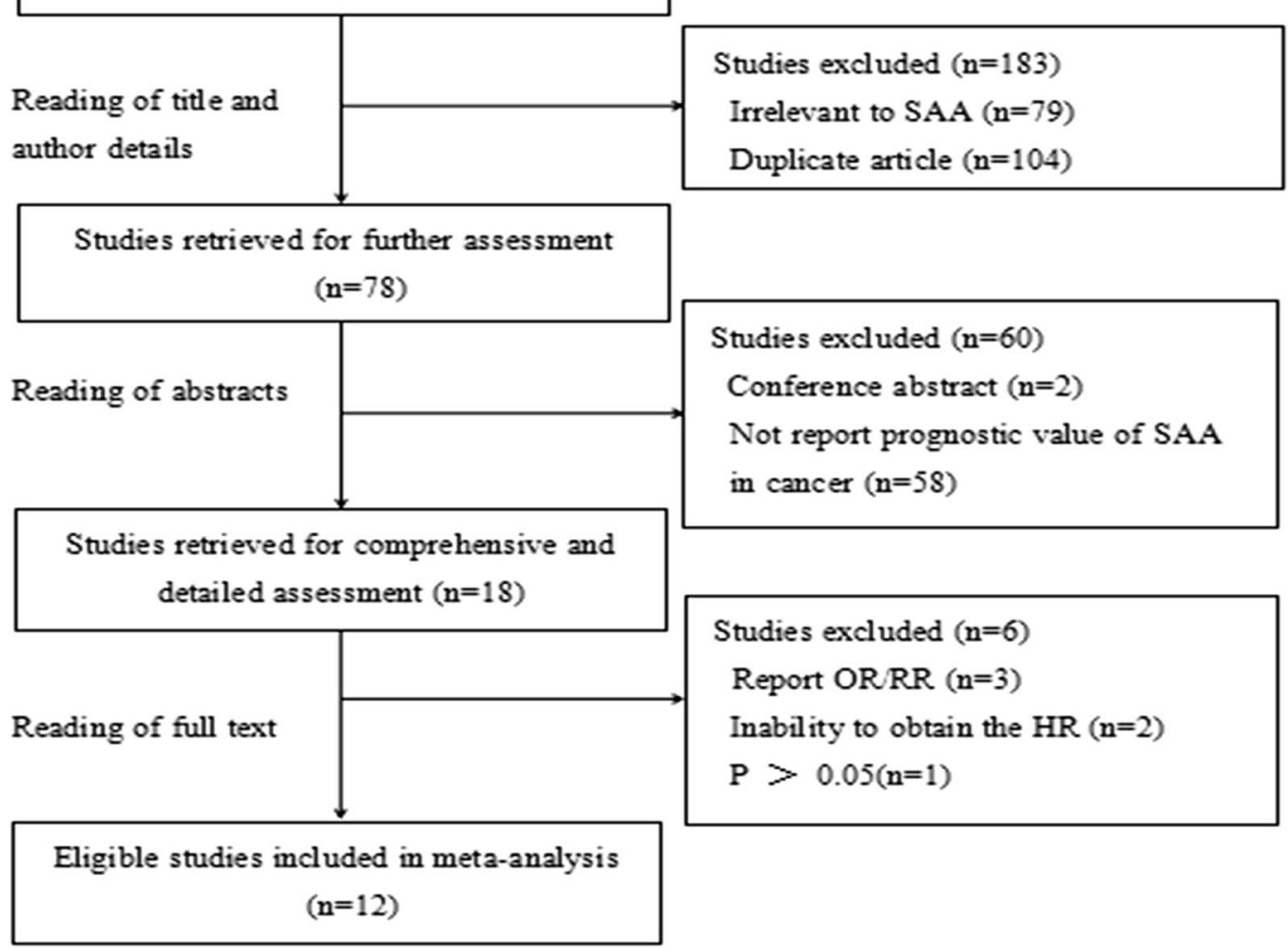

Fig. 1 The flow chart of the study selection process in this meta-analysis

\section{Characteristics of the studies}

In total, 12 eligible studies with 2749 patients published between 2000 and 2017 were included in the present meta-analysis. Seven studies were conducted in Asian countries (China, Japan and South Korea), and 5 were conducted in non-Asian countries (USA, UK, Holland and Germany). Nine different types of cancer were involved in the eligible studies, namely, renal cell carcinoma, breast cancer, gastric cancer, esophageal squamous cell carcinoma, rectal cancer, hepatocellular carcinoma, pancreatic cancer, nasopharyngeal carcinoma and non-small cell lung. The level of SAA in these studies was mostly measured using the nephelometry method, while enzyme-linked immunosorbent assays (ELISA) and latex agglutination turbidimetric immunoassays (LTIA) were also utilized. The cut-off value for the level of SAA was reported in 11 studies, and it ranged from $19.2 \mathrm{ng} / \mathrm{ml}$ to $22.0 \mathrm{mg} / \mathrm{l}$. Among the included studies, OS was reported in 12 studies, and DFS/PFS was reported in 3 studies. The results of the studies were analysed by multivariate or univariate methods. Furthermore, only one study required extrapolation of the HR for OS, and the HRs and 95\% CIs for both OS and DFS/PFS were directly reported in the other studies. The quality assessment scores of the included studies ranged from 5 to 9 , all of which were regarded as high quality (Table 1 ). The detailed characteristics of the included studies are shown in Table 2.

\section{Correlation between SAA level and survival outcome}

Twelve studies reported the relationship between SAA level and OS in a total of 2749 cancer patients. For OS, we calculated a pooled HR using a random effects model because significant heterogeneity was observed in this meta-analysis $\left(I^{2}=82.7 \%, P=0.000\right)$. The pooled HR for OS was 3.01 (95\% CI 1.96-4.63, $P<0.01$ ), which suggested that an elevated level of SAA was significantly associated with poor OS in cancer patients (Fig. 2). Three studies reported the relationship between the SAA level and DFS/PFS in a total of 1003 cancer patients. For DFS/PFS, we calculated the pooled HR using a fixed effects model because no heterogeneity was observed in this meta-analysis $\left(I^{2}=0.0 \%, P=0.912\right)$. The pooled HR for DFS/PFS was 1.67 (95\% CI 1.31-2.12, $P<0.01$ ), which also suggested that an elevated level of SAA was 


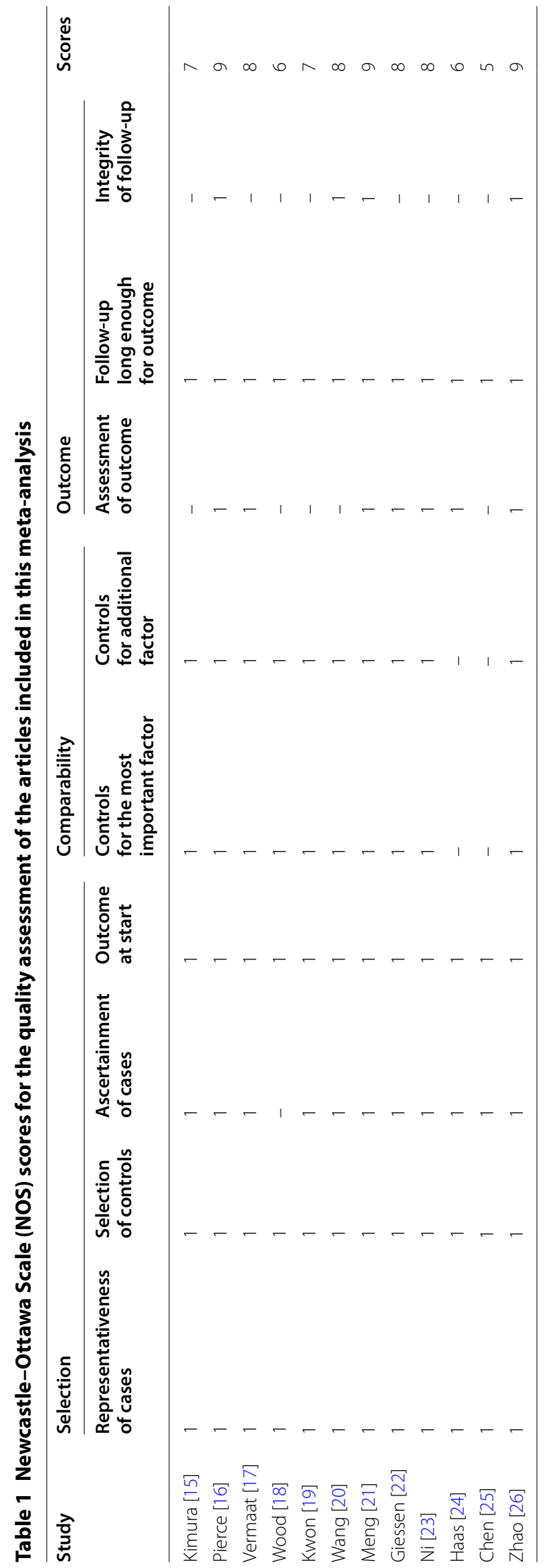




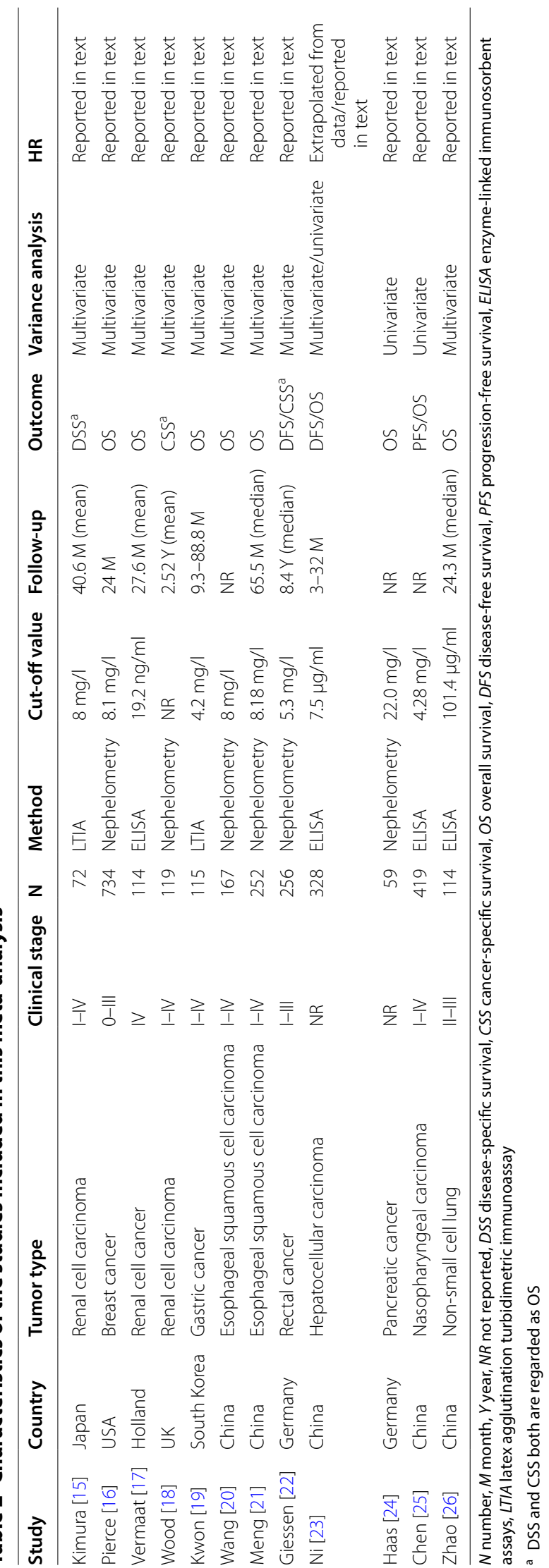




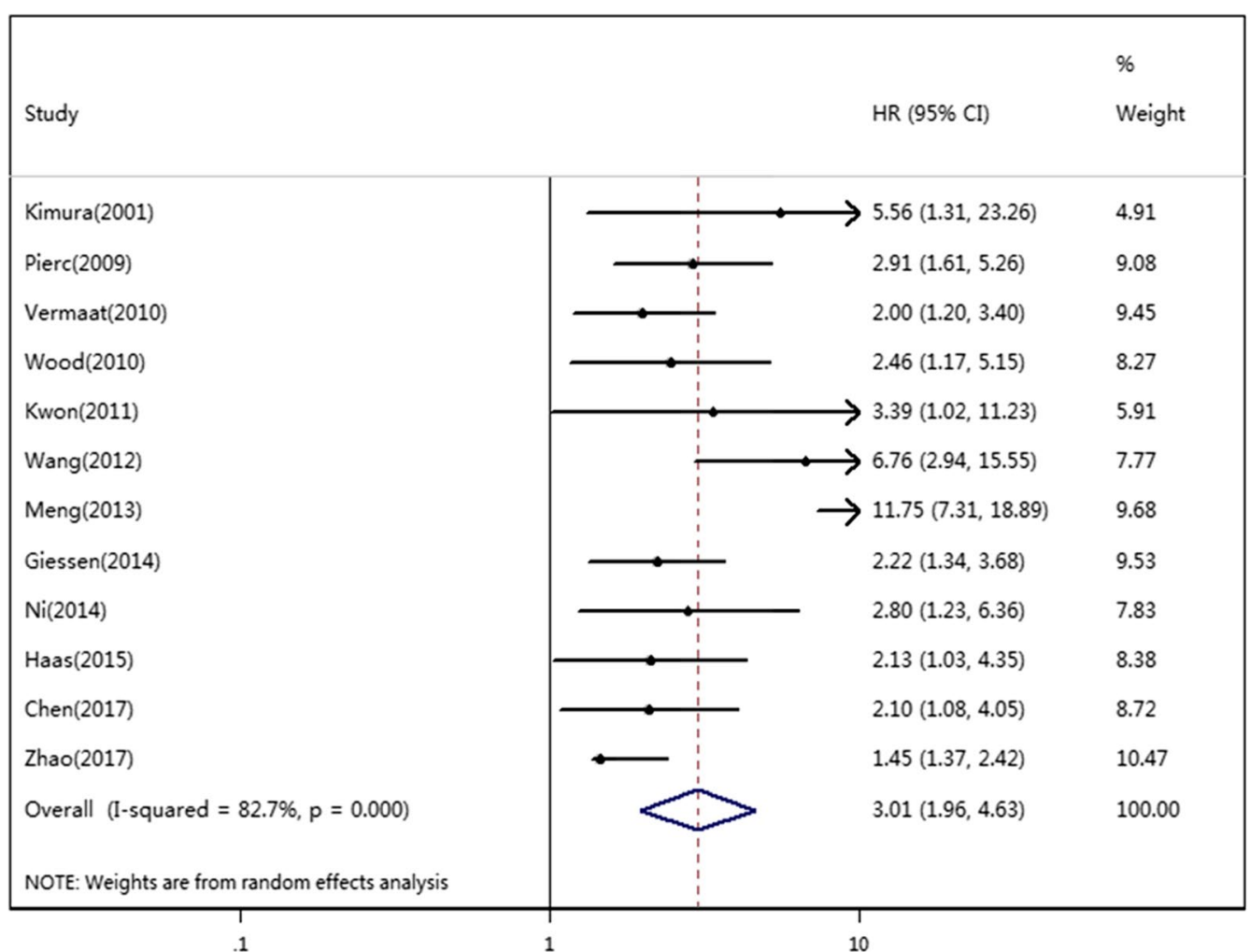

Fig. 2 Forest plots of pooled HR of the relationship between SAA level and OS

significantly associated with poor DFS/PFS in cancer patients (Fig. 3).

\section{Publication bias and sensitivity analysis}

For the OS, publication bias was revealed by asymmetrical funnel plots (Fig. 4). The result of Egger test's was not significant $(P=0.214)$, but Begg's test was significant $(P=0.047)$. Based on the trim and fill analysis for OS, no missing studies were imputed in the contourenhanced funnel plots (Fig. 5). The analysis indicated that the imputed HR was 3.02 (95\% CI 1.96-4.63), which had no influence on the overall effect of SAA level on OS. To assess whether the results were reliable, it was necessary to conduct further sensitivity analysis. After the removal of any single included study, the sensitivity analysis showed no significant change in the pooled estimates of the influence of the SAA level on the OS of patients with solid tumors (Fig. 6). For the DFS/PFS, the sample sizes were too small to conduct sensitivity and publication bias analyses.

\section{Subgroup and meta-regression analyses}

For the OS, subgroup and meta-regression analyses were performed according to tumor types, detection methods, cut-off values, sample sizes, areas, and variance analyses.
In the analysis stratified by tumor type, elevated levels of SAA were significantly correlated with poor OS in urinary cancers $(\mathrm{HR}=2.31,95 \% \mathrm{CI} 1.54-3.48)$, digestive system cancers $(\mathrm{HR}=3.97,95 \% \mathrm{CI} 1.98-7.94)$ and other cancers ( $\mathrm{HR}=1.70,95 \% \mathrm{CI} 1.34-2.16)$. When different detection methods were considered, elevated levels of SAA were significantly correlated with poor OS in the LTIA group ( $\mathrm{HR}=4.15,95 \% \mathrm{CI} 1.65-10.43)$, the nephelometry group $(\mathrm{HR}=3.75,95 \% \mathrm{CI} 1.97-7.14)$ and the ELISA group $(\mathrm{HR}=1.69,95 \% \mathrm{CI} 1.35-2.11)$. In the subgroup analysis by cut-off value, we found that poor OS was significantly correlated with a cut-off value less than or equal to the median value of $5.3 \mathrm{mg} / \mathrm{l}(\mathrm{HR}=1.80$, $95 \% \mathrm{CI} 1.47-2.20)$ and greater than $5.3 \mathrm{mg} / \mathrm{l}(\mathrm{HR}=4.87$, 95\% CI 2.32-10.26). A similar result was also observed in the subgroup analysis by sample size, both sample sizes less than or equal to a median value of $143(\mathrm{HR}=1.77$, 95\% CI 1.42-2.20) and less than $143(\mathrm{HR}=3.82$, 95\% CI 2.01-7.25) were significantly associated with OS. In addition, we also observed that elevated levels of SAA were associated with poor OS in studies conducted in Asian countries (HR $=3.78,95 \% \mathrm{CI} 1.72-8.32)$ and in nonAsian countries ( $\mathrm{HR}=2.30,95 \%$ CI $1.76-3.00)$. Moreover, an elevated level of SAA was significantly associated with poor $\mathrm{OS}$ in multivariate analysis $(\mathrm{HR}=3.32,95 \% \mathrm{CI}$ 


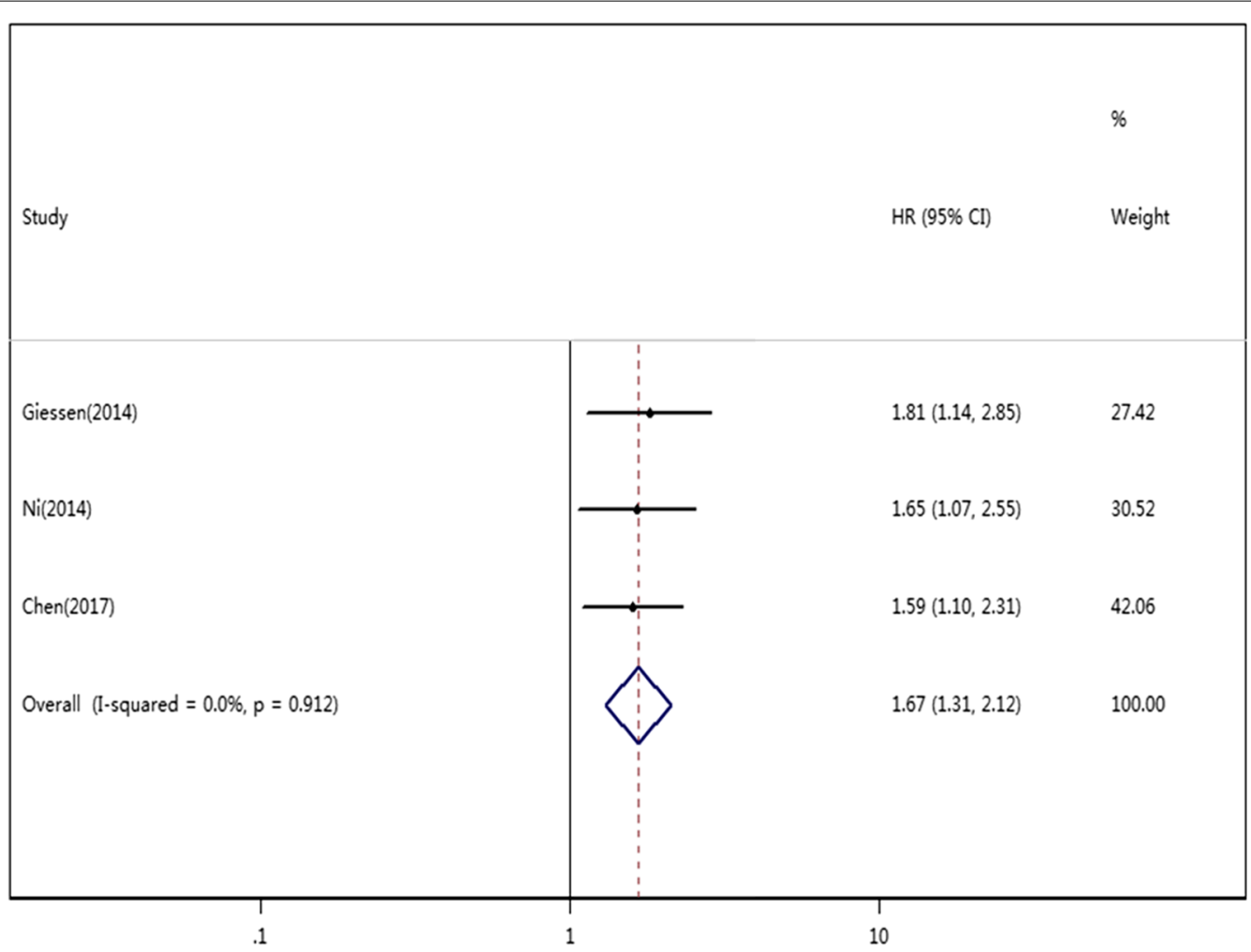

Fig. 3 Forest plots of pooled HR of the relationship between SAA level and DFS/PFS

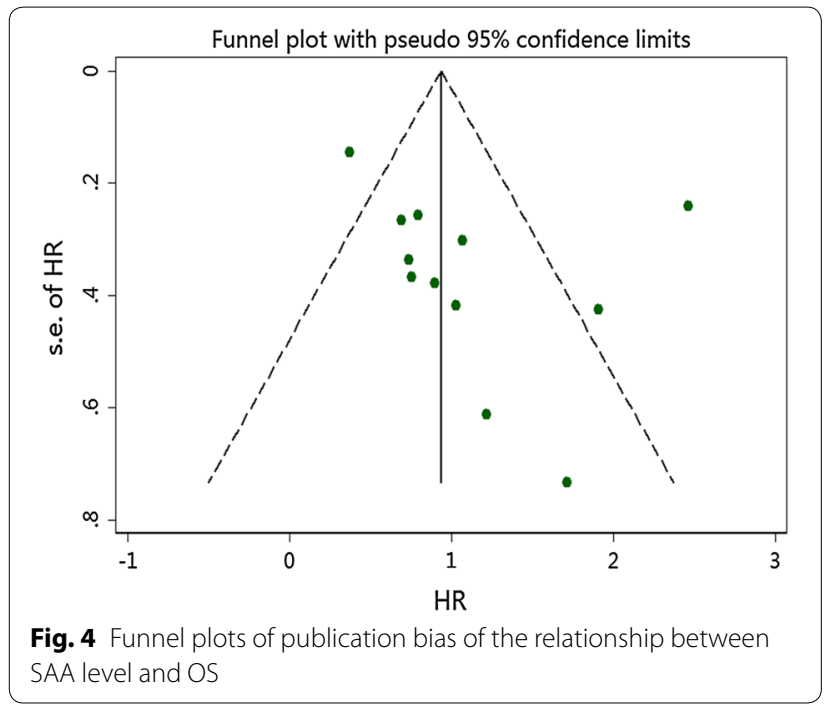

1.91-5.77) and univariate analysis $(\mathrm{HR}=2.27,95 \% \mathrm{CI}$ 1.50-3.45). More importantly, meta-regression analysis found that cut-off value had statistically significant in the inter-study heterogeneity $(P=0.03)$, indicating that cutoff value might help explain the sources of the inter-study

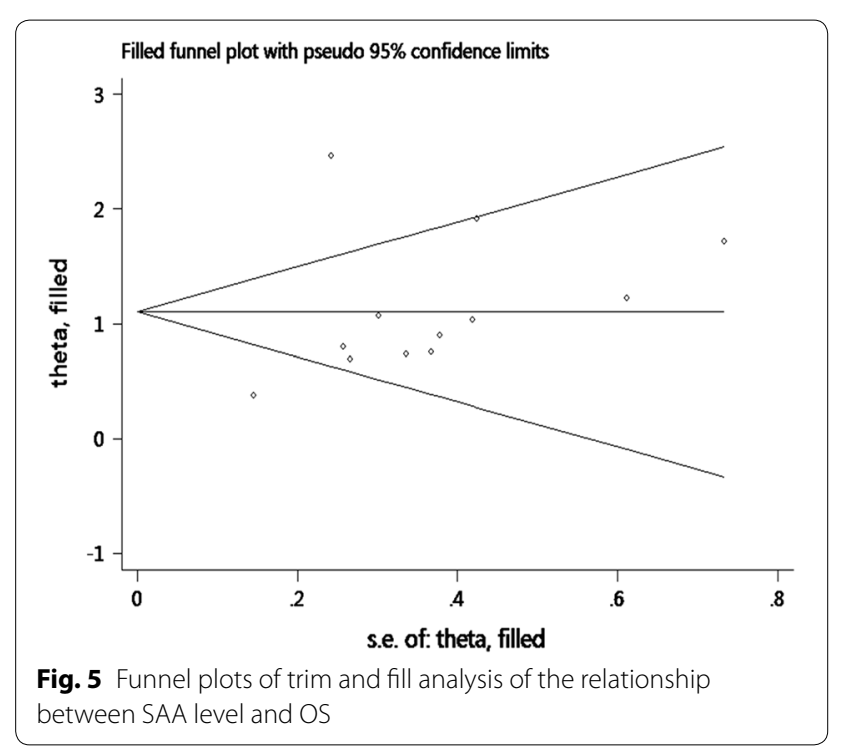

heterogeneity. All results of the subgroup and metaregression analyses for OS are shown in Table 3. However, For the DFS/PFS, the sample sizes were too small to conduct subgroup and meta-regression analyses. 


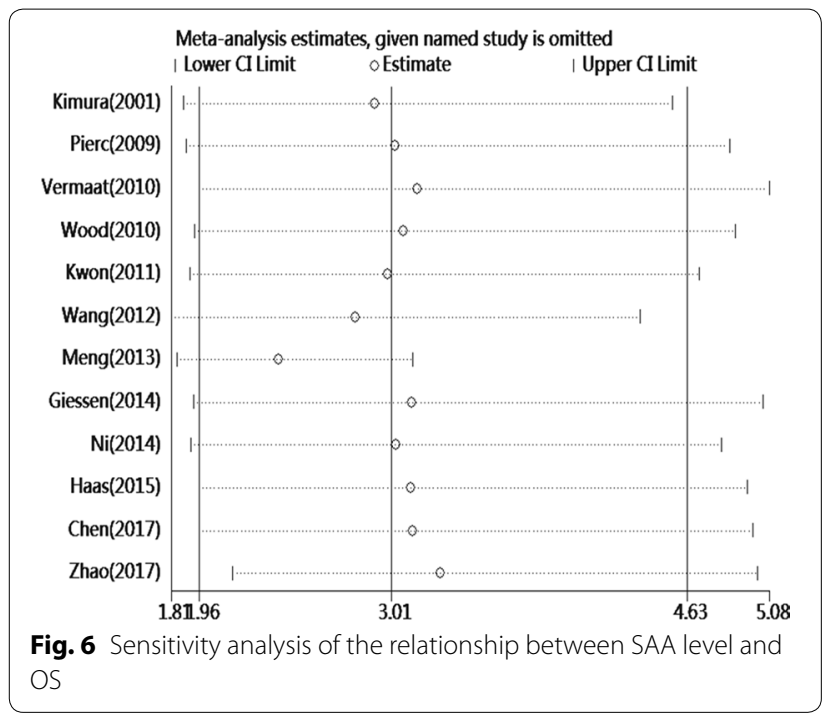

\section{Discussion}

SAA is an acute-phase protein mainly produced by the liver under the regulation of inflammation-associated cytokines in the course of acute and chronic inflammatory processes. However, SAA is also synthesized in extrahepatic tissues, including primary and metastatic cancer cell lines [27, 28]. Previous studies have shown that SAA is an ideal biomarker for monitoring inflammation in many types of cancer [29]. Moreover, the sensitivity of SAA for the detection of the inflammatory response is considered to be tenfold higher than that of CRP [30]. Accumulated evidence demonstrates that tumor development is closely associated with chronic infection and inflammation. In 1979, there was already evidence that an elevated level of SSA was found in cancer patients [31]. Subsequently, SAA was proposed as a possible serum biomarker for many cancers, including renal cell cancer, breast cancer, gastric cancer, oesophageal cancer, rectal cancer, hepatocellular cancer, pancreatic cancer, and nasopharyngeal carcinoma [15-20, 22-25]. Clearly, SAA serves as a possible link between chronic inflammation and tumourigenesis, and elevated levels of SAA could contribute to tumor development and accelerate tumor progression and metastasis. However, because cancer is often considered a consequence of chronic inflammation, the consensus among many researchers is that SAA might influence tumor invasion through the extracellular matrix (ECM) by stimulating the production of matrix metalloproteinases (MMPs) [32]. Furthermore, SAA can modulate platelet adhesion and influence the adhesion of tumor cells to platelets,

Table 3 Subgroup and meta-regression analyses for OS in this meta-analysis

\begin{tabular}{|c|c|c|c|c|c|c|c|}
\hline \multirow[t]{2}{*}{ Subgroup } & \multirow[t]{2}{*}{ No. of studies } & \multirow[t]{2}{*}{ No. of patients } & \multicolumn{2}{|c|}{ Fixed-effects model } & \multirow{2}{*}{$\begin{array}{l}\text { Meta-regression } \\
P \text { value }\end{array}$} & \multicolumn{2}{|c|}{ Heterogeneity } \\
\hline & & & HR $(95 \% \mathrm{Cl})$ & $P$-value & & $P^{2}(\%)$ & $P$-value \\
\hline All & 12 & 2749 & $3.01(1.96-4.63)$ & $<0.01$ & & 82.7 & 0.00 \\
\hline Tumor types & & & & & 0.30 & & \\
\hline Urinary system cancer & 3 & 305 & $2.31(1.54-3.48)$ & $<0.01$ & & 0.0 & 0.42 \\
\hline Digestive system cancer & 6 & 1177 & $3.97(1.98-7.94)$ & $<0.01$ & & 82.3 & 0.00 \\
\hline Other system cancer & 3 & 1267 & $1.70(1.34-2.16)$ & $<0.01$ & & 57.9 & 0.09 \\
\hline Detection methods & & & & & 0.24 & & \\
\hline LTIA & 2 & 187 & $4.15(1.65-10.43)$ & $<0.01$ & & 0.0 & 0.61 \\
\hline Nephelometry & 6 & 1587 & $3.75(1.97-7.14)$ & $<0.01$ & & 84.5 & 0.00 \\
\hline ELISA & 4 & 975 & $1.69(1.35-2.11)$ & $<0.01$ & & 11.2 & 0.34 \\
\hline Cut-off values (mg/l) & & & & & 0.03 & & \\
\hline$\leq 5.3$ & 6 & 1346 & $1.80(1.47-2.20)$ & $<0.01$ & & 7.9 & 0.37 \\
\hline$>5.3$ & 5 & 1284 & $4.87(2.32-10.26)$ & $<0.01$ & & 81.1 & 0.00 \\
\hline Sample sizes & & & & & 0.16 & & \\
\hline$\leq 143$ & 6 & 593 & $1.77(1.42-2.20)$ & $<0.01$ & & 25.0 & 0.25 \\
\hline$>143$ & 6 & 2156 & $3.82(2.01-7.25)$ & $<0.01$ & & 84.4 & 0.00 \\
\hline Areas & & & & & 0.26 & & \\
\hline Asian countries & 7 & 1467 & $3.78(1.72-8.32)$ & $<0.01$ & & 90.3 & 0.00 \\
\hline Non-Asian countries & 5 & 1282 & $2.30(1.76-3.00)$ & $<0.01$ & & 0.0 & 0.91 \\
\hline Variance analyses & & & & & 0.46 & & \\
\hline Multivariate & 9 & 1943 & $3.32(1.91-5.77)$ & $<0.01$ & & 87.3 & 0.00 \\
\hline Univariate & 3 & 806 & $2.27(1.50-3.45)$ & $<0.01$ & & 0.0 & 0.84 \\
\hline
\end{tabular}


which may contribute to tumor invasion [32]. The precise mechanisms underlying the association of a high level of SAA with the development and progression of cancer are still poorly understood. Moreover, the prognostic role of SAA in solid tumors remains uncertain and needs to be addressed.

To the best of our knowledge, our study is the first meta-analysis assessing the prognostic role of SAA in various solid tumors. The meta-analysis included a total of 2749 patients with solid tumors, including renal cell carcinoma, breast cancer, gastric cancer, esophageal squamous cell carcinoma, rectal cancer, hepatocellular carcinoma, pancreatic cancer, nasopharyngeal carcinoma and non-small cell lung. In this meta-analysis, by estimating the pooled HR of the included studies, an elevated level of SAA was found to be significantly related to poor OS and DFS/PFS in patients with solid tumors. Although publication bias for OS was found in the meta-analysis, the results after adjustment by the trim and fill method were consistent with the original results. For the OS, further sensitivity analysis demonstrated that the result was not affected after excluding any single study. Finally, subgroup analysis between SAA levels and OS were performed, and an elevated level of SAA was still a negative marker for OS when the patients were stratified by tumor types, detection methods, cut-off values, sample sizes, areas, and variance analyses. Although inter-study heterogeneity was not significantly decreased in the subgroup analysis stratified by several factors, meta-regression analysis subsequently found that cut-off value maybe an important source of heterogeneity. Based on the above evidence, the results of our study are stable and reliable.

When stratified by tumor type, there was a trend for the association of increased SAA levels with poor OS to be the most sensitive for solid tumors in the digestive system, as the pooled HR of the subgroup with digestive system cancer was the highest of the entire group. However, this finding was not reported by previous studies. A similar result was observed in the subgroup analysis by cut-off value, the relationship between increased SAA level and poor OS was more sensitive for the subgroup with $>5.3 \mathrm{mg} / \mathrm{l} \mathrm{SAA}$ than the subgroup with $\leq 5.3 \mathrm{mg} / \mathrm{l}$ SAA. This indicates that a greater tumor burden may aggravate the inflammatory response. Compared with ELISA, nephelometry showed greater sensitivity in predicting OS in the subgroup analyses by detection methods. Certainly, there is a need to confirm our results with further clinical trials.

However, some limitations in the current meta-analysis need to be acknowledged. First, only English language articles were included, while articles written in other languages were excluded. Second, one article could not directly provide a HR and its $95 \% \mathrm{CI}$. Thus, we extracted the $\mathrm{HR}$ and $95 \% \mathrm{CI}$ through the procedure recommended by Tierney et al. [13], which may result in small statistical errors. Third, some studies provided HRs and 95\% CIs from univariable analyses, which could lead to bias towards overestimation of the prognostic role of SAA, as the HRs in multivariable analyses may not be statistically significant after the consideration of other elements. Furthermore, the sample size of the included articles that reported DFS/PFS were too small to conduct publication bias, sensitivity, subgroup and meta-regression analyses, which might influence the stability of the corresponding results. Therefore, much more evidences need to confirm SAA can effectively predict DFS/PFS of patients with solid tumors. Finally, twelve studies in our meta-analysis both reported elevated levels of SAA are significantly associated with outcome in patients solid tumors. Latest article suggested that SAA maybe considered as a potential molecule to monitor the progression of acute lymphoblastic leukaemia [33]. However, until now, there are rare studies reported the relation between SAA and prognosis of patients with hematological malignancies. Thus we further recommend more clinical trials to determine prognostic value of SAA in hematological malignancies.

\section{Conclusion}

In conclusion, the current meta-analysis showed that elevated levels of SAA are significantly associated with poor OS and DFS/PFS in patients with solid tumos. Furthermore, SAA might be used as a novel biomarker to predict OS of patients with solid tumors. In the future, larger-scale, multicentre and prospective studies are needed to validate our conclusions.

\section{Authors' contributions}

Conceptualization: SL, HL, GQ. Data analysis: HL, GQ, LY. Original draft writing: HL, GQ, SL. Review and editing: SL, HL, GQ, LY. All authors read and approved the final manuscript.

\section{Author details}

${ }^{1}$ Department of Orthopedics, The Third Affiliated Hospital of Southern Medical University, Guangzhou 510630, Guangdong, China. ${ }^{2}$ Department of Oncology, Jiangmen Central Hospital, Jiangmen 529030, Guangdong, China.

${ }^{3}$ Department of Oncology, Affiliated Dongfeng Hospital, Hubei University of Medicine, Shiyan 442008, Hubei, China. ${ }^{4}$ Clinical Department of Guangdong Metabolic Disease Research Center of Integrated Chinese and Western Medicine, The First Affiliated Hospital of Guangdong Pharmaceutical University, Guangzhou 510080, Guangdong, China.

\section{Acknowledgements}

We would like to thank all researchers for their contributions.

Competing interests

The authors declare that they have no competing interests.

Availability of data and materials

The databases analyzed during the current study are available. 


\section{Consent for publication}

Not applicable.

\section{Ethics approval and consent to participate}

The present meta-analysis was based on previous published studies, and no experiments involving humans and animal. Therefore, no ethical approval are required.

\section{Funding}

The present study was supported by the National Natural Science Foundation of China (No. 81071751).

\section{Publisher's Note}

Springer Nature remains neutral with regard to jurisdictional claims in published maps and institutional affiliations.

Received: 9 December 2018 Accepted: 15 March 2019

Published online: 20 March 2019

\section{References}

1. Torre LA, Bray F, Siegel RL, et al. Global cancer statistics, 2012. CA A Cancer J Clin. 2015:65(2):87-108.

2. Shimada H, Noie T, Ohashi M, et al. Clinical significance of serum tumor markers for gastric cancer: a systematic review of literature by the Task Force of the Japanese Gastric Cancer Association. Gastric Cancer. 2014;17(1):26-33.

3. Coussens LM, Werb Z. Inflammation and cancer. Nature. 2002;420(6917):860-7.

4. Philip M, Rowley DA, Schreiber H. Inflammation as a tumor promoter in cancer induction. Semin Cancer Biol. 2004;14(6):433-9.

5. Candido J, Hagemann T. Cancer-related inflammation. J Clin Immunol. 2013;33(Suppl 1):S79-84.

6. Schultz DR, Arnold PI. Properties of four acute phase proteins: C-reactive protein, serum amyloid A protein, alpha 1-acid glycoprotein, and fibrinogen. Semin Arthritis Rheum. 1990;20(3):129-47.

7. Kushner I. The acute phase response: an overview. Methods Enzymol. 1988;163:373-83.

8. Moshkovskii SA. Why do cancer cells produce serum amyloid A acutephase protein? Biochemistry. 2012;77(4):339-41.

9. Huang Y, Feng JF, Liu JS, et al. Prognostic role of serum C-reactive protein in esophageal cancer: a systematic review and meta-analysis. Ther Clin Risk Manag. 2015:11:89-94.

10. Woo HD, Kim K, Kim J. Association between preoperative C-reactive protein level and colorectal cancer survival: a meta-analysis. Cancer Causes Control CCC. 2015;26(11):1661-70.

11. Zhou L, Cai X, Liu Q, et al. Prognostic role of C-reactive protein in urological cancers: a meta-analysis. Sci Rep. 2015;5:12733. https://doi. org/10.1038/srep12733.

12. Zhou J, Sheng J, Fan Y, et al. Association between serum amyloid A levels and cancers: a systematic review and meta-analysis. Postgrad Med J. 2018:94(1115):499-507.

13. Tierney JF, Stewart LA, Ghersi D, et al. Practical methods for incorporating summary time-to-event data into meta-analysis. Trials. 2007;8:16. https:// doi.org/10.1186/1745-6215-14-391.

14. Duval S, Tweedie R. Trim and fill: a simple funnel-plot-based method of testing and adjusting for publication bias in meta-analysis. Biometrics. 2000;56(2):455-63.

15. Kimura $M$, Tomita $Y$, Imai T, et al. Significance of serum amyloid $A$ on the prognosis in patients with renal cell carcinoma. Cancer. 2001;92(8):2072-5.
16. Pierce $B L$, Ballard-Barbash $R$, Bernstein L, et al Elevated biomarkers of inflammation are associated with reduced survival among breast cancer patients. J Clin Oncol. 2009:27(21):3437-44.

17. Vermaat JS, van der Tweel I, Mehra N, et al. Two-protein signature of novel serological markers apolipoprotein-A2 and serum amyloid alpha predicts prognosis in patients with metastatic renal cell cancer and improves the currently used prognostic survival models. Ann Oncol. 2010;21(7):1472-81.

18. Wood SL, Rogers M, Cairns DA, et al. Association of serum amyloid A protein and peptide fragments with prognosis in renal cancer. Br J Cancer. 2010;103(1):101-11.

19. Kwon H, Kim SH, Oh SY, et al. Clinicopathologic significance of expression of nuclear factor kappa B and its target gene products in gastric cancer patients. World J Gastroenterol. 2012;18(34):4744-50.

20. Wang JY, Zheng YZ, Yang J, et al. Elevated levels of serum amyloid $A$ indicate poor prognosis in patients with esophageal squamous cell carcinoma. BMC Cancer. 2012;23(12):365. https://doi. org/10.1186/1471-2407-12-365

21. Meng YQ, Cao X, Wen ZS, et al. Preoperative level of serum amyloid A is superior to C-reactive protein in the prognosis of esophageal squamous cell carcinoma. Dis Esophagus. 2014;27(7):670-7.

22. Giessen C, Nagel D, Glas M, et al. Evaluation of preoperative serum markers for individual patient prognosis in stage I-III rectal cancer. Tumour Biol. 2014;35(10):10237-48.

23. Ni XC, Yi Y, Fu YP, et al. Serum amyloid A is a novel prognostic biomarker in hepatocellular carcinoma. Asian Pac J Cancer Prev: APJCP. 2014:15(24):10713-8

24. Haas M, Kern C, Kruger S, et al. Assessing novel prognostic serum biomarkers in advanced pancreatic cancer: the role of CYFRA 21-1, serum amyloid A, haptoglobin, and 25-OH vitamin D3. Tumour Biol. 2015:36(4):2631-40.

25. Chen QY, Tang QN, Tang LQ, et al. Pretreatment serum amyloid $A$ and C-reactive protein comparing with Epstein-Barr Virus DNA as prognostic indicators in patients with nasopharyngeal carcinoma: a prospective study. Cancer Res Treat. 2017. https://doi.org/10.4143/crt.2017.180.

26. Zhao J, Li X, Zhao X, et al. Study on the correlation of serum amyloid A level with overall survival and radiation pneumonitis in non-small cell lung cancer patients receiving thoracic radiotherapy. Precis Radiat Oncol. 2017;1(2):46-51.

27. Parle-McDermott A, McWilliam $P$, Tighe $O$, et al. Serial analysis of gene expression identifies putative metastasis-associated transcripts in colon tumour cell lines. Br J Cancer. 2000:83(6):725-8.

28. Djurec M, Grana O, Lee A, et al. Saa3 is a key mediator of the protumorigenic properties of cancer-associated fibroblasts in pancreatic tumors. Proc Natl Acad Sci USA. 2018;115(6):E1147-56.

29. Malle E, De Beer FC. Human serum amyloid A (SAA) protein: a prominent acute-phase reactant for clinical practice. Eur J Clin Invest. 1996;26(6):427-35.

30. Yamada T. Serum amyloid A (SAA): a concise review of biology, assay methods and clinical usefulness. Clin Chem Lab Med. 1999;37(4):381-8.

31. Rosenthal CJ, Sullivan LM. Serum amyloid A to monitor cancer dissemination. Ann Intern Med. 1979;91(3):383-90.

32. Malle E, Sodin-Semrl S, Kovacevic A. Serum amyloid A: an acutephase protein involved in tumour pathogenesis. Cell Mol Life Sci. 2009:66(1):9-26.

33. Raza SK, Shamsi T, Musharraf SG. Serum amyloid A1 and plasminogen as predictory proteins to monitor the progression of preleukemic diseases towards acute lymphoblastic leukaemia. RSC Adv. 2017;7(46):29218-26. 